\title{
Characterization of Surface Acoustic Wave Optomechanical Oscillators
}

\author{
Gaurav Bahl, John Zehnpfennig, Matthew Tomes, and Tal Carmon \\ Optics and Photonics, Electrical Engineering and Computer Science \\ University of Michigan \\ Ann Arbor, Michigan, USA 48105 \\ Email: bahlg@umich.edu
}

\begin{abstract}
We describe and experimentally demonstrate an optomechanical oscillator where light drives a surface mechanical mode of a spherical resonator, using a combination of photoelastic scattering and optical electrostriction. These oscillators are shown to have discretely-selectable frequencies ranging from $50 \mathrm{MHz}$ to $1.5 \mathrm{GHz}$ on a single silica microsphere device. We also report on initial measurements of phase noise and continuous frequency tuning of these oscillators.
\end{abstract}

\section{INTRODUCTION}

In recent years, oscillators based on parametric mechanical vibration in optical microresonators have emerged as promising candidates for compact and low power photonic frequency references [1]-[4]. While prior work has focused on the use of radiation pressure and gradient forces [5] in order to drive mechanical vibration, here we exploit optical electrostriction [6] to stimulate (as in a laser) an acoustical whispering gallery resonance of the microsphere. These acoustical modes are concentrated at the equator of the sphere, and have been calculated through finite element modeling to be shear type and Rayleigh type [7] surface acoustic waves (SAW).

Such SAW-based optomechanical oscillators (OMO) have been theoretically proposed in the past [8]. In this new class of optomechanical oscillator, incoming light drives and interacts with the surface acoustic mode, resulting in two optical signals at the output of the device (Figure 1(a)). The beat note between these optical signals is measured with a photodetector, and is at the acoustical frequency of the surface mode that we excited.

\section{Surface Acoustic Wave Optomechanical OSCILLATORS}

We will now describe the process of exciting optomechanical oscillations within these spherical resonators. An incoming pump photon scatters from an acoustic phonon occupying a mechanical eigenmode of the spherical resonator, resulting in a scattered Stokes photon that is down-shifted to a lower frequency. The scattered Stokes light together with the incoming pump light generates electrostrictive pressure which amplifies the initial acoustic wave. Energy and momentum are conserved in this process through the generation of phonons at the same acoustical frequency from which the pump light was initially scattered. The process is illustrated in Figure 1(b) and is similar to stimulated Brillouin scattering that takes place in bulk materials [6].

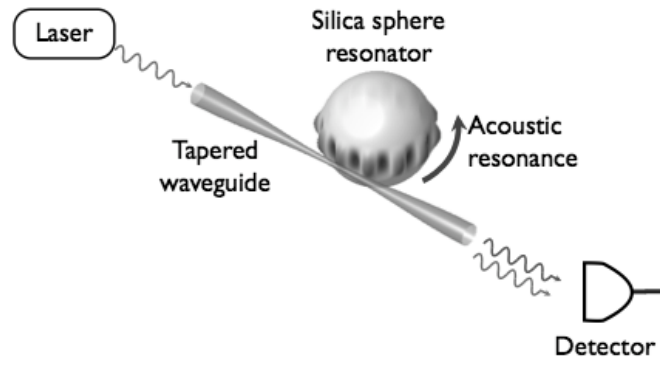

(a) Schematic of the oscillator. The detector outputs the beat note between the two optical signals, which is at the mechanical frequency.

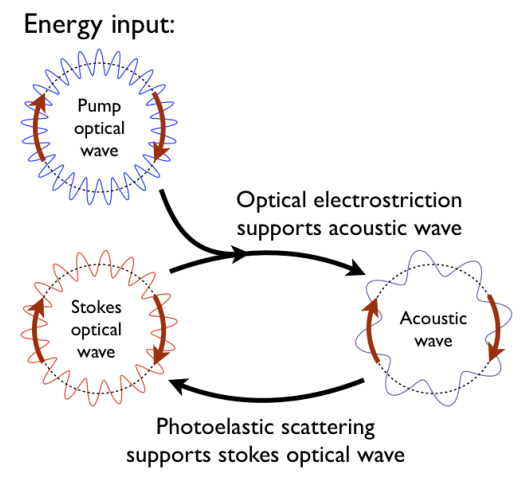

(b) Optical electrostriction and photoelastic scattering drive the mechanical oscillations of a traveling acoustical mode

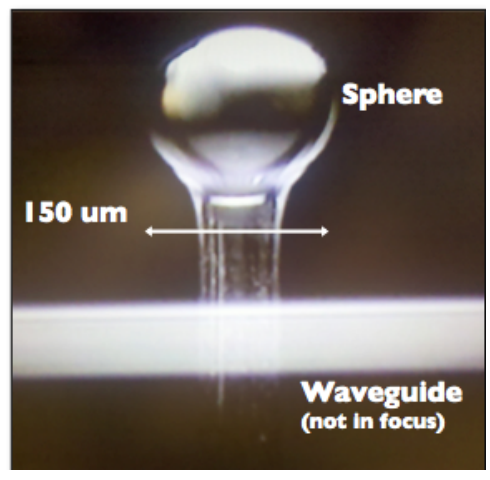

(c) Microscope image of the oscillator

Fig. 1. Surface acoustic wave optomechanical oscillator: Theory and practical realization 
The experimental realization of a SAW-OMO (Figure 1(a)) requires two optical whispering gallery modes that have extremely close frequencies (in relative optical terms) but at the same time have different wavevectors. This requirement can be expressed as follows

$$
\begin{aligned}
M_{P} & =M_{S}+M_{A} \\
\omega_{P} & =\omega_{S}+\omega_{A} .
\end{aligned}
$$

Here the $M_{i}$ parameters are the number of periods of the optical modes ('P', ' $\mathrm{S}$ ' subscripts) and of the acoustical mode ('A' subscript) around the circumference of the sphere, and their relationship describes momentum conservation. The simultaneous frequency relationship $\omega_{i}$ describes energy conservation. This phase matching requirement is the result of solving for a synchronous solution [9] to the optical and acoustical wave equations that describe the processes of photoelastic scattering and optical electrostriction within the sphere. Light is coupled in and out of the microsphere resonator [10] using a tapered optical fiber [11]-[13]. Optical quality factors are in the range of $10^{8}$.

Previous demonstrations of oscillations induced via Brillouin scattering, shown in $\mathrm{CaF}_{2}$ [14] and in silica [15], depended on back-scattering from the acoustical wave to close the internal feedback loop in the above described process. When we consider momentum conservation in back-scattering, the resulting phonons have a wavelength that is approximately half of the pump light used. For a 1.5 um wavelength pump propagating in silica, this translates to an optical wavelength of $1 \mathrm{um}$ within the resonator, and an acoustical wavelength of $0.5 \mathrm{um}$ resulting in frequencies in the $11 \mathrm{GHz}$ range [15] for when silica is the host material. However, the lifetime for such hypersonic phonons is extremely short, resulting in low acoustical finesse. The acoustical frequencies are primarily dictated by material properties in this case.

A question arises whether lower frequencies, dictated to some extent by geometry, can be targeted instead. Lower frequency phonons would have much longer lifetimes within the host material, and would have a much higher acoustical finesse within the microsphere. As opposed to previous demonstrations of backward scattering, here we rely on forward scattering [8], [16] to generate lower frequency phonons. Momentum conservation for forward scattering dictates that the acoustic frequency is significantly smaller, and is of the order of $100 \mathrm{MHz}$.

\section{IDENTIFICATION OF MECHANICAL MODES}

Previous OMO demonstrations, have primarily been based on mechanical parametric oscillation of optical microcavities. The standard varying parameter in these cases is the optical path length and the mechanical modes can be described as breathing modes (Figure 2(a)). The resulting optical spectrum at the output thus shows modulation effects (phase and amplitude) with sidebands at both higher and lower frequencies. Higher frequency harmonics of these sidebands are also present [4].

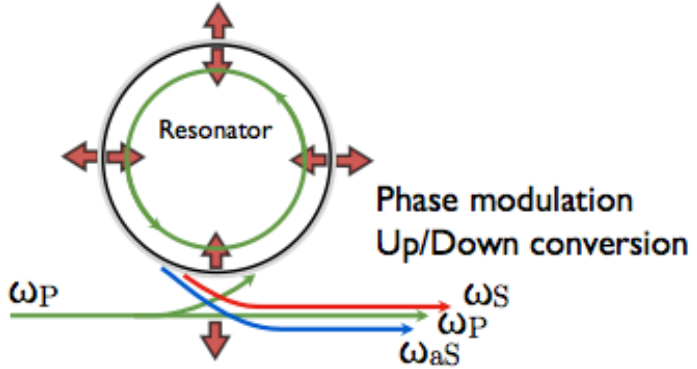

(a) Breathe modes generate both upper and lower sidebands (with higher harmonics) to the optical input.

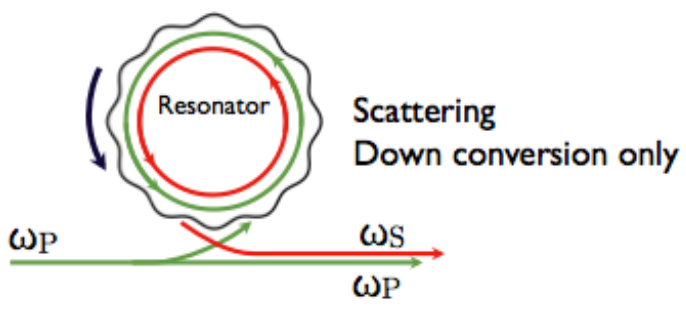

(b) Surface acoustic modes only generate a single reddownshifted sideband.

Fig. 2. Differentiating mechanical modes through optical output signals

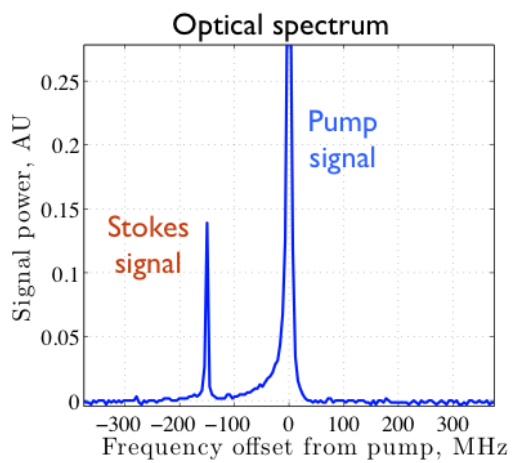

Fig. 3. Verification of the surface-wave nature of a $150 \mathrm{MHz}$ oscillation. Only one Stokes red-shifted optical line is present adjacent to the pump.

In the case described in this work, the mechanical excitation is a surface mode that travels unidirectionally along the equator (Figure 2(b)). Light that is scattered from this traveling mode is always downshifted from the pump due to the always-receding nature of the mode. The optical path length does not change. As a result, there is only a single Stokes-frequency optical sideband that appears at the output.

Since there is a difference between the optical signatures of the two types of mechanical modes, we can distinguish between them by resolving the spectra of the output light using an optical spectrum analyzer. We have experimentally studied the optical spectra to confirm the generation of surface mechanical modes (Figure 3). 


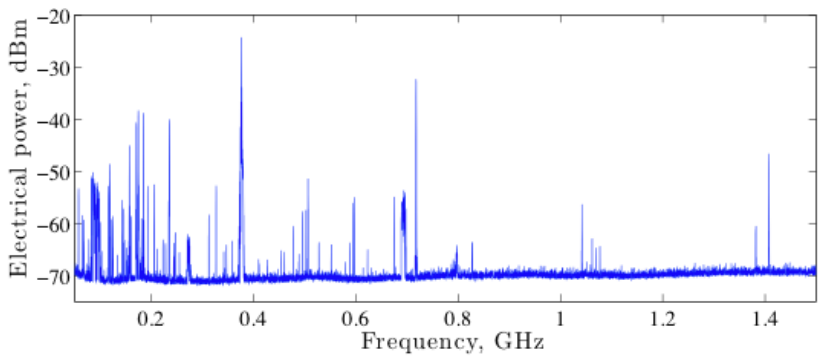

(a) Many low frequency oscillations $(50 \mathrm{MHz}-1.4 \mathrm{GHz})$ can be excited (this work)

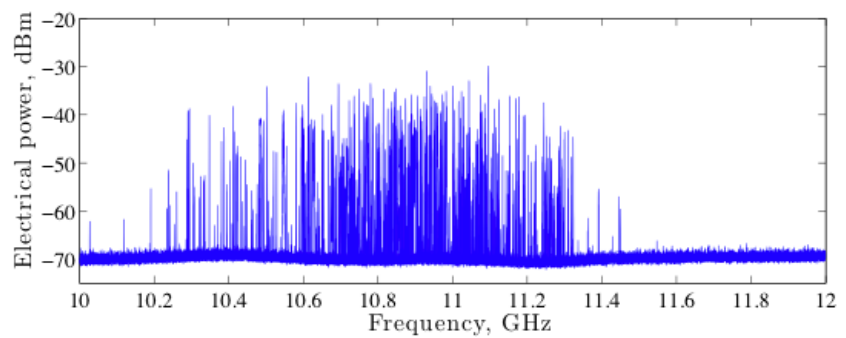

(b) High frequency oscillations around $11 \mathrm{GHz}$ can also be excited (prior art)

Fig. 4. Excitation of various oscillation frequencies while slowly scanning the pump laser from $1520-1570 \mathrm{~nm}$.

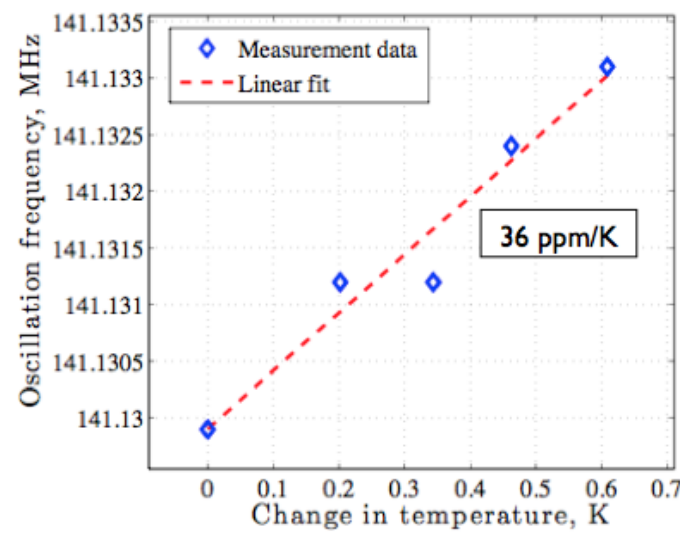

Fig. 5. The capability for continuous frequency tuning using temperature exists. The tuning slope for a given oscillation depends on the specific combination of optical and mechanical modes that participate in the process. The pictured measurement is for a $141 \mathrm{MHz}$ oscillation.

\section{EXPERIMENTAL RESULTS}

\section{A. Oscillation frequency and tuning}

We have experimentally determined (Figure 4) that a single device can show hundreds of discretely-selectable oscillation frequencies through the described process. In the experiment shown in Figure 4(a), we scanned the pump laser from 1520 $\mathrm{nm}-1570 \mathrm{~nm}$ in order to probe many pump modes, while keeping the electrical spectrum analyzer on the 'peak hold' setting. In the low frequency regime that this paper describes, we were able to capture oscillations ranging from $50 \mathrm{MHz}$ up to $1.4 \mathrm{GHz}$.

We can combine this result with the previously demon-

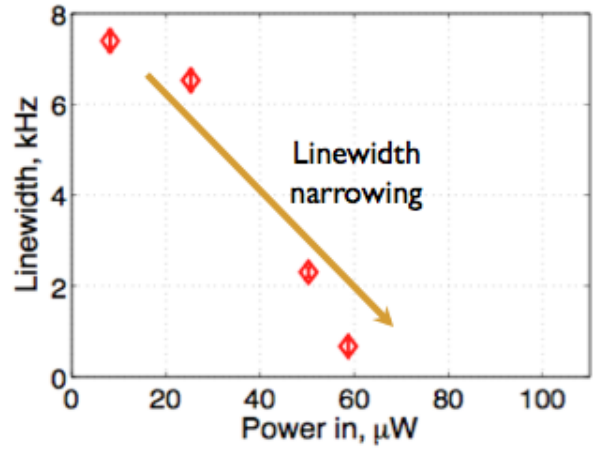

Fig. 6. Oscillation linewidth narrows with increasing optical pump power, consistent with the narrowing of laser linewidth.

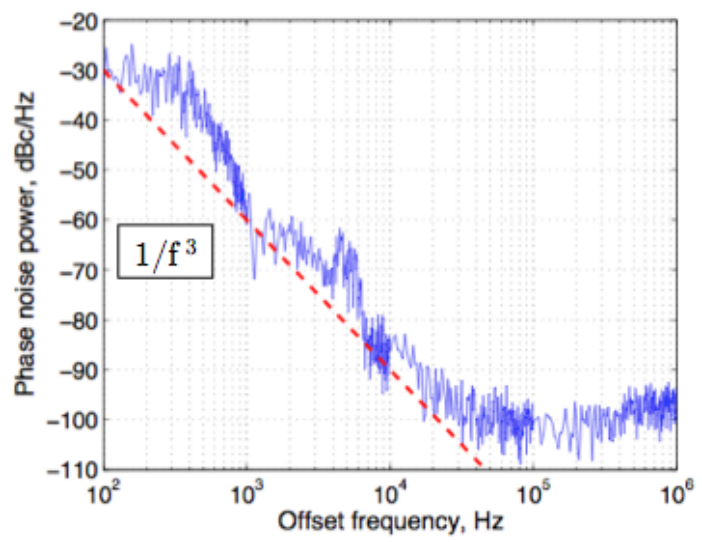

Fig. 7. Initial phase noise measurements indicate a $1 / f^{3}$ slope. The phase noise measurement from a $94 \mathrm{MHz}$ oscillation at $-35 \mathrm{dBm}$ signal power is pictured here.

strated ability to excite acoustical modes in the X-band $(11.5 \mathrm{GHz})$ frequency regime via Brillouin back-scattering (Figure 4(b)). The implications of this combined result are that a single device can produce oscillations ranging from $50 \mathrm{MHz}-1.4 \mathrm{GHz}$, and also from $10 \mathrm{GHz}-11.5 \mathrm{GHz}$. Indeed there is a large gap between these frequency ranges where oscillations have not been experimentally demonstrated. We can, however, begin to fill this gap by using different pump wavelengths and different materials.

Beyond the capability for discrete frequency selection with this oscillator, we can also thermally tune the oscillation frequency (Figure 5). Here, the major effect responsible for tuning is the positive temperature coefficient of material stiffness for silica. The tunability however does appear to depend on the specific mechanical and optical modes that participate in any given oscillation. It must be noted that temperature change also affects the optical modes.

\section{B. Noise performance}

We have performed several experiments studying the noise performance of these oscillators. Our data shows narrowing of the oscillation linewidth as a function of increasing pump optical power (Figure 6). The linewidth narrowing is consistent 
with lasing processes. This line narrowing behavior should continue until the system reaches the Schawlow-Townes limit. We also have preliminary phase noise measurements which indicate a $1 / f^{3}$ characteristic close to carrier (Figure 7).

\section{CONCLUSIONS}

We have experimentally demonstrated a class of optomechanical oscillators that excite surface acoustic waves on silica microspheres through electrostrictive pressure generated by light. While SAW are typically generated on planar piezoelectric substrates, with metal electrodes used for applying electric fields, here we apply the electric field through a set of virtual electrodes formed by light which co-propagates with the traveling acoustical mode. Additionally, the excitation mechanism of electrostriction is available in any dielectric material, opening up broad possibilities in material selection. Since SAW-based sensors are well-studied, the potential applications of this technology extend beyond oscillators into various sensing techniques.

\section{REFERENCES}

[1] T. Carmon, H. Rokhsari, L. Yang, T. Kippenberg, and K. Vahala, "Temporal behavior of radiation-pressure-induced vibrations of an optical microcavity phonon mode," Phys. Rev. Lett., vol. 94, no. 22, p. 223902, 2005.

[2] H. Rokhsari, T. Kippenberg, T. Carmon, and K. Vahala, "Radiationpressure-driven micro-mechanical oscillator," Opt. Express, vol. 13, no. 14, pp. 5293-5301, Jul 2005.

[3] T. J. Kippenberg, H. Rokhsari, T. Carmon, A. Scherer, and K. J. Vahala, "Analysis of radiation-pressure induced mechanical oscillation of an optical microcavity," Phys. Rev. Lett., vol. 95, no. 3, p. 033901, Jul 2005.

[4] T. Carmon and K. J. Vahala, "Modal Spectroscopy of Optoexcited Vibrations of a Micron-Scale On-Chip Resonator at Greater than $1 \mathrm{GHz}$ Frequency," Phys. Rev. Lett., vol. 98, no. 12, p. 123901, Mar 2007.

[5] J. Rosenberg, Q. Lin, and O. Painter, "Static and dynamic wavelength routing via the gradient optical force," Nature Photonics, vol. 3, pp. 478 -483 , July 2009.

[6] R. W. Boyd, Nonlinear Optics, 3rd ed. Elsevier, 2008, Chapter 9.

[7] L. Rayleigh, "On waves propagated along the plane surface of an elastic solid," Proc. R. Soc. Lond., vol. A17, pp. 4 - 11, 1885.

[8] A. B. Matsko, A. A. Savchenkov, V. S. Ilchenko, D. Seidel, and L. Maleki, "Optomechanics with surface-acoustic-wave whisperinggallery modes," Phys. Rev. Lett., vol. 103, no. 25, p. 257403, Dec 2009.

[9] H. A. Haus and W. Huang, "Coupled-mode theory," Proceedings of the IEEE, vol. 79, no. 10, pp. $1505-1518$, Oct. 1991.

[10] M. Gorodetsky and V. Ilchenko, "High-Q optical whispering-gallery microresonators: precession approach for spherical mode analysis and emission patterns with prism couplers," Optics Communications, vol. 113, no. $1-3$, pp. 133 - 143, 1994.

[11] M. Cai and K. Vahala, "Highly efficient hybrid fiber taper coupled microsphere laser," Opt. Lett., vol. 26, no. 12, pp. 884-886, Jun 2001.

[12] S. Spillane, T. Kippenberg, O. Painter, and K. Vahala, "Ideality in a Fiber-Taper-Coupled Microresonator System for Application to Cavity Quantum Electrodynamics," Phys. Rev. Lett., vol. 91, no. 4, p. 043902, Jul 2003.

[13] J. C. Knight, G. Cheung, F. Jacques, and T. A. Birks, "Phase-matched excitation of whispering-gallery-mode resonances by a fiber taper," Optics Letters, vol. 22, no. 15, pp. 1129-1131, 1997.

[14] I. S. Grudinin, A. B. Matsko, and L. Maleki, "Brillouin lasing with a $\mathrm{CaF}_{2}$ whispering gallery mode resonator," Phys. Rev. Lett., vol. 102, no. 4, p. 043902, Jan 2009.

[15] R. Y. Chiao, C. H. Townes, and B. P. Stoicheff, "Stimulated brillouin scattering and coherent generation of intense hypersonic waves," Phys. Rev. Lett., vol. 12, no. 21, pp. 592-595, May 1964.

[16] R. Shelby, M. Levenson, and P. Bayer, "Resolved forward Brillouin scattering in optical fibers," Physical review letters, vol. 54, no. 9, pp. 939-942, 1985. 